\title{
Trigger primitive generation algorithm in the CMS barrel muon chambers during HL-LHC
}

\section{Nicolò Trevisani, for the CMS Collaboration}

University of Oviedo - ICTEA,

Calle San Francisco, 3, 33003, Oviedo, Asturias (Spain)

E-mail: nicolo.trevisani@cern.ch

This paper presents an update on the Analytical Method algorithm for trigger primitive generation in the CMS Drift Tube chambers during the High Luminosity LHC operation. The algorithm has been developed and validated both in software with an emulation approach, and through hardware implementation tests. The algorithm is mainly divided into the following steps: a grouping (pattern recognition) step that finds the path of a given muon, a fitting step to extract the track parameters (position and bending angle), and a correlation step that matches the information from the different super-layers with the signal from the Resistive Plate Chambers. Agreement between the software emulation and the firmware implementation has been verified using different data samples, including a sample of real muons collected during 2016 data taking. This paper also includes an update on the merging of grouping and correlation steps.

\footnotetext{
*** The European Physical Society Conference on High Energy Physics (EPS-HEP2021), *** ***26-30 July $2021 * * *$

*** Online conference, jointly organized by Universität Hamburg and the research center DESY ***
} 


\section{Introduction}

The Compact Muon Solenoid (CMS) is one of the two multi-purpose experiments at the Large Hadron Collider (LHC). It includes a two-level trigger system: the Level-1 Trigger (L1), implemented in custom-designed electronics, and the High-Level Trigger (HLT), a streamlined version of the CMS offline reconstruction software [1]. The outermost part of the experimental apparatus hosts muon detectors, with drift tubes (DTs) in the barrel and cathode strip chambers (CSCs) covering the endcaps, both complemented by resistive plate chambers (RPCs). The fundamental element of a drift tube is the drift cell, which reconstructs hits with a left-right ambiguity in their position (laterality). Four staggered layers of parallel cells form a super-layer (SL), which provides the measurement of two-dimensional segments. A chamber contains two super-layers measuring the $\mathrm{r}-\phi$ coordinate and an orthogonal super-layer measuring the r-z coordinate [2]. The High-Luminosity phase of the LHC (HL-LHC) will start after 2027, reaching an instantaneous luminosity of $7.5 \times 10^{34} \mathrm{~cm}^{-2} \mathrm{~s}^{-1}$ [3]. A total replacement of DT electronics will take place to cope with the new environment, and Field Programmable Gate Arrays (FPGAs) will perform trigger primitives generation [3].

\section{The Analytical Method for DT Trigger Primitive Generation at HL-LHC}

The Analytical Method (AM) is an algorithm designed to perform DT trigger primitive generation at HL-LHC, reconstructing the muon collision time ( $\left.\mathrm{t}_{0}\right)$, bunch crossing (BX), global position $(\phi)$, and direction (bending $\phi)$. The inputs of the AM are the IDs of the drift cells that have registered signals and the hits timestamps with respect to the start of the LHC orbit, while it has to make assumptions on the hits lateralities. The algorithm works in four steps [3]:

- Grouping: in each super-layer, the AM selects signals in patterns of 4 cells compatible with straight lines and the corresponding sub-patterns of 3 cells. For each pattern, it then assigns a set of lateralities to be considered as candidates in the fitting step;

- Fitting: the algorithm uses analytical expressions to obtain $t_{0}$ and then the muon track parameters ( $\phi$ and bending $\phi)$ from a $\chi^{2}$ minimization fit;

- Correlation: patterns with 3 or 4 hits in both SL1 and SL3 can be correlated if the corresponding reconstructed segment times agree within a window of $25 \mathrm{~ns}$. The track parameters are then updated, considering the original positions and collision times in SL1 and SL3.

- Cleaning: if candidates share hits, the AM keeps those with higher quality.

The AM can also consider timing information from the RPCs to refine the $\mathrm{t}_{0}$ estimation.

\section{Algorithm Performance and Validation}

The AM efficiency and resolution are measured using samples simulating the HL-LHC conditions. The results presented use offline reconstruction as a reference, and scenarios with and without detector ageing are studied. Efficiencies, shown in Figure 1a, are very close to 1, and RPCs help in 
maintaining performance in case of detector ageing. Resolutions in global position $\phi$ and direction $\phi_{B}$ are presented in Figure 1c and $1 \mathrm{~d}$ and are less than about $0.05 \mathrm{mrad}$ and $1 \mathrm{mrad}$, respectively, representing a significant improvement with respect to Phase-1 results [3]. The algorithm has been implemented both in software and in firmware to run on test FPGAs. Figure $1 \mathrm{~b}$ shows the almost perfect agreement between the two approaches [3], obtained using the AM on data collected during proton-proton collisions in 2016.

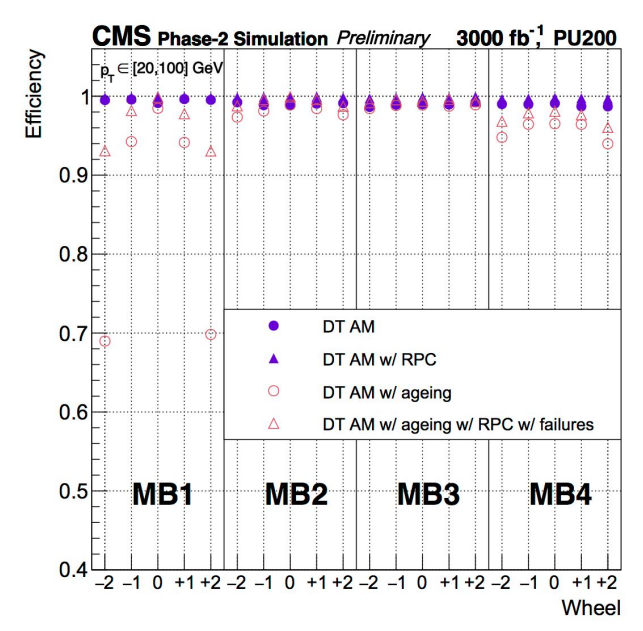

(a)

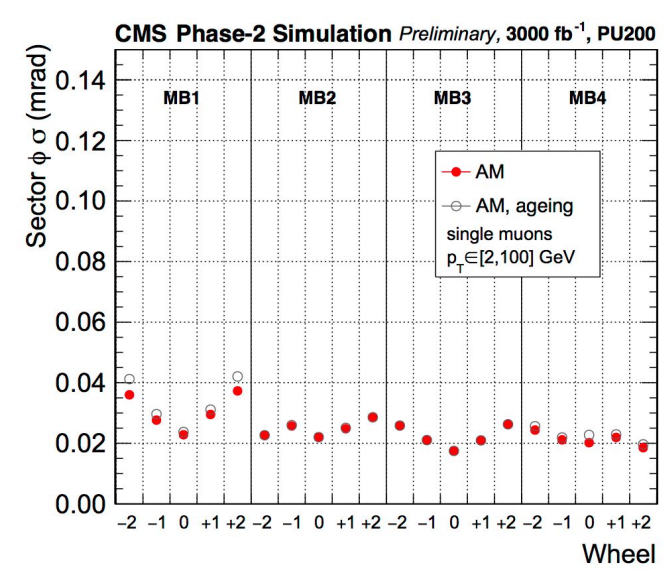

(c)

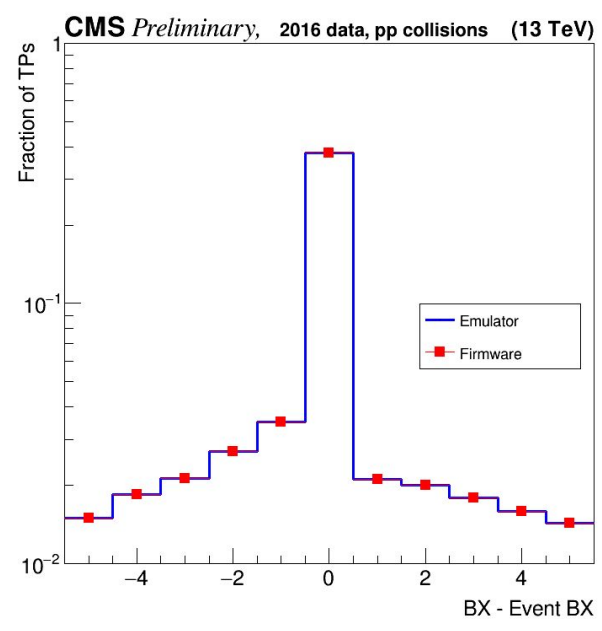

(b)

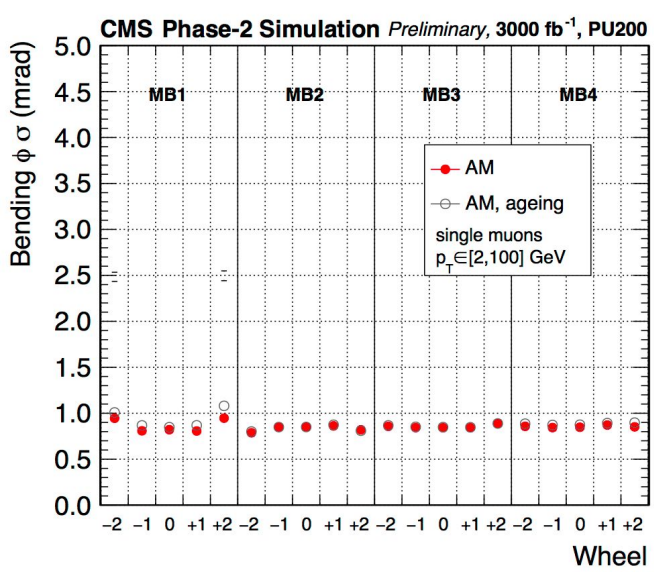

(d)

Figure 1: Top row: efficiency of the AM algorithm with respect to offline reconstruction (a) and difference between the BX assigned by the AM algorithm and the event BX (b), when using emulator (blue) and firmware (red). Bottom row: global position resolution (c) and bending angle resolution (d) with respect to the simulated hits in the muon chambers

\section{Merging of Grouping and Correlation Steps}

One alternative strategy to the grouping step is under study. It considers hits from both $\mathrm{r}-\phi$ super-layers, using a set of pre-computed patterns (straight lines, due to the low magnetic field in 
the chambers [4]), which represent the possible trajectories of muons crossing the DT chambers and include information on the predicted laterality of every hit. Some expected advantages are:

- higher resilience in severe ageing scenarios;

- reduced noise due to fewer candidates;

- fewer combinations of hits to test, as the correlation between super-layers is introduced already at the grouping stage

Finally, the fitting step receives the patterns selected at the grouping stage, extracting the track parameters from a single $\chi^{2}$ minimization involving a maximum of 8 hits.

\section{Conclusions}

The Analytical Method is an algorithm designed to perform DT trigger primitive generation on FPGAs during HL-LHC. Tests performed both in software and firmware, using data and simulated samples tuned to reproduce the Phase- 2 conditions, show efficiencies close to 1 and resolutions that significantly improve those provided by the Phase- 1 algorithm. Possible refinements of the current design are under study and include correlation between super-layers at the grouping stage to increase resilience in case of extreme ageing and to reduce the number of muon candidates.

\section{References}

[1] The CMS Collaboration and S Chatrchyan et al., The CMS experiment at the CERN LHC, JINST 3 (2008) S08004

[2] CMS Collaboration, The CMS muon project: Technical Design Report, CERN (1997)

[3] CMS Collaboration, The Phase-2 Upgrade of the CMS Level-1 Trigger, CERN (2020)

[4] CMS Collaboration, CMS Physics: Technical Design Report Volume 1: Detector Performance and Software, CERN (2006) 